\title{
Wissenswertes
}

\section{zu Wissensplattformen}

\author{
Jürg Fröhlicha, Dominique Froidevaux ${ }^{\mathrm{b}}$, Romeo Providoli ${ }^{\mathrm{c}}$ \\ ${ }^{a}$ Dr. med., Facharzt Allgemeine Innere Medizin, Mitglied Wissenschaftliches Komitee «in a nutshell»; ${ }^{b}$ M Sc, Inhaber Pro Medicus GmbH; \\ 'Dr. med., Facharzt Allgemeine Innere Medizin und Sportmedizin, SGAIM-Vorstandsmitglied, Mitglied Wissenschaftliches Komitee «in a nutshell»
}

\begin{abstract}
Hausärztinnen und Hausärzte sind für viele Patienten und Patientinnen die erste Anlaufstelle. Als Grundversorger decken sie praktisch das gesamte Spektrum der modernen Medizin ab und begleiten ihre Patienten und Patientinnen meist über viele Jahre. Oft ist die Konsultationsdauer knapp bemessen, und es mangelt an Zeit für fundierte Recherchen. Wissensplattformen helfen, die benötigten Informationen rasch und präzise abzurufen.
\end{abstract}

\section{Herausforderungen in der Grundversorgung}

Aktuell verdoppelt sich das medizinische Wissen fünfmal (!) pro Jahr [1]. Laufend werden neue Studienerkenntnisse und Guidelines publiziert. Für Hausärzte, die ja «überall» kompetent sein sollen, wird dies zunehmend zur Herausforderung.

Wie soll die Ärzteschaft dem Vertrauen, das in sie gesetzt wird, auch künftig gerecht werden? Welchen Impact hat der demographische Wandel in der Bevölkerung (Verdoppelung des Alterssegments der 70-80-Jährigen) [2] und bei den Ärzten und Ärztinnen (50\% der Hausarztpraxen schliessen in den nächsten 10 Jahren) [3]? Wie werden modifizierte Curricula, neue Tarifmodelle, zunehmende Bürokratie und die vermehrte Teilzeitarbeit den Erfahrungsschatz und die Zeitbudgets der Hausärztinnen und Hausärzte beeinflussen?

\section{Ein Blick in die Zukunft}

promedicus.ch

Tel. 0432669917

Pro Medicus GmbH

Bahnhofplatz 4

CH-8001 Zürich

d.froidevaux[at]promedicus.ch
Was in der gesamten Arbeitswelt gilt, kommt auch in der Medizin zum Tragen: Kollaborative Ansätze, Digitalisierung, Vernetzung. Gerade der COVID-19-Lockdown hat uns alle gelehrt, wie wichtig und nützlich solche Tools sind.

\section{Wissensplattformen - der Mehrwert für den Praxisalltag}

Grundversorgerinnen und Grundversorger bilden sich regelmässig an Fortbildungen aller Art kontinuierlich weiter. Was bis dato gefehlt hat, ist ein niederschwelliger Zugang zu Top-Experten und -Expertinnen in jedem Fachgebiet und ein zentraler Ort, an welchem der Arzt / die Ärztin alle Informationen und Hilfsmittel abholen kann, und zwar: rasch, evidenzbasiert, immer verfügbar, wenn man's braucht («on demand») und stets aktualisiert. Genau dies bieten moderne Wissensplattformen.

Charakteristisch für moderne Wissensplattformen ist, dass sie sich an den Informationsbedürfnissen ihrer Zielgruppe orientieren (konkreter Praxisbezug, rasch auffindbare Informationen und Hilfsmittel) und dass sie evidenzbasiert und fachlich breit abgestützt sind. Wissensplattformen können wesentlich dazu beitragen, dass ...

... sich Hausärzte und Hausärztinnen untereinander und mit Spezialisten und Spezialistinnen stärker vernetzen;

... Ärztinnen und Ärzte weniger Zeit verlieren bei der Suche und Verwendung von praxisorientiertem Wissen; .. ein Beitrag geleistet wird für eine nachhaltige Qualitätsentwicklung. 